\title{
Association of Irritability and Anxiety With the Neural Mechanisms of Implicit Face Emotion Processing in Youths With Psychopathology
}

Joel Stoddard, MD; Wan-Ling Tseng, PhD; Pilyoung Kim, PhD; Gang Chen, PhD; Jennifer Yi, BA; Laura Donahue, BS; Melissa A. Brotman, PhD; Kenneth E. Towbin, MD; Daniel S. Pine, MD; Ellen Leibenluft, MD

IMPORTANCE Psychiatric comorbidity complicates clinical care and confounds efforts to elucidate the pathophysiology of commonly occurring symptoms in youths. To our knowledge, few studies have simultaneously assessed the effect of 2 continuously distributed traits on brain-behavior relationships in children with psychopathology.

OBJECTIVE To determine shared and unique effects of 2 major dimensions of child psychopathology, irritability and anxiety, on neural responses to facial emotions during functional magnetic resonance imaging.

DESIGN, SETTING, AND PARTICIPANTS Cross-sectional functional magnetic resonance imaging study in a large, well-characterized clinical sample at a research clinic at the National Institute of Mental Health. The referred sample included youths ages 8 to 17 years, 93 youths with anxiety, disruptive mood dysregulation, and/or attention-deficit/hyperactivity disorders and 22 healthy youths.

MAIN OUTCOMES AND MEASURES The child's irritability and anxiety were rated by both parent and child on the Affective Reactivity Index and Screen for Child Anxiety Related Disorders, respectively. Using functional magnetic resonance imaging, neural response was measured across the brain during gender labeling of varying intensities of angry, happy, or fearful face emotions. In mixed-effects analyses, the shared and unique effects of irritability and anxiety were tested on amygdala functional connectivity and activation to face emotions.

RESULTS The mean (SD) age of participants was 13.2 (2.6) years; of the 115 included, 64 were male. Irritability and/or anxiety influenced amygdala connectivity to the prefrontal and temporal cortex. Specifically, irritability and anxiety jointly influenced left amygdala to left medial prefrontal cortex connectivity during face emotion viewing $\left(F_{4,888}=9.20 ; P<.001\right.$ for mixed model term). During viewing of intensely angry faces, decreased connectivity was associated with high levels of both anxiety and irritability, whereas increased connectivity was associated with high levels of anxiety but low levels of irritability (Wald $\chi^{2}{ }_{1}=21.3$; $P<.001$ for contrast). Irritability was associated with differences in neural response to face emotions in several areas $\left(F_{2,888} \geq 13.45\right.$; all $\left.P<.001\right)$. This primarily occurred in the ventral visual areas, with a positive association to angry and happy faces relative to fearful faces.

CONCLUSIONS AND RELEVANCE These data extend prior work conducted in youths with irritability or anxiety alone and suggest that research may miss important findings if the pathophysiology of irritability and anxiety are studied in isolation. Decreased amygdala-medial prefrontal cortex connectivity may mediate emotion dysregulation when very anxious and irritable youth process threat-related faces. Activation in the ventral visual circuitry suggests a mechanism through which signals of social approach (ie, happy and angry expressions) may capture attention in irritable youth.

JAMA Psychiatry. 2017;74(1):95-103. doi:10.1001/jamapsychiatry.2016.3282

Published online November 30, 2016.
Supplemental content

Author Affiliations: Author affiliations are listed at the end of this article.

Corresponding Author: Joel Stoddard, MD, University of Colorado, 13123 E 16th Ave, Box A036/B130, Aurora, CO 80045 (joel.stoddard@ucdenver.edu). 
T he Research Domain Criteria (RDoC) framework calls for studies examining the neural circuitry of dimensional traits across diagnoses. Such studies are particularly important in children, who typically present with impairment due to symptoms spanning multiple diagnoses and dimensions. Thus, while previous studies have examined the neural circuitry mediating single symptom dimensions, it is important to extend this work by examining interactions among 2 or more commonly co-occurring traits. Here, we test the hypothesis that dimensional variation in irritability and anxiety jointly influence the neural circuitry of face emotion processing.

Irritability and anxiety are 2 of the most common, frequently co-occurring problems of youth seeking psychiatric care. Research reveals strong clinical and pathophysiological associations between them. Longitudinal studies have shown that childhood irritability predicts the risk for anxiety in adulthood, ${ }^{1-3}$ whereas cognitive studies have found similar attention biases in youths with irritability and anxiety. ${ }^{4-6}$ However, virtually no research has considered how anxiety and irritability independently and mutually predict brain function.

Independent lines of research have linked irritability and anxiety to perturbed amygdala-prefrontal cortex (PFC) circuitry function during face emotion processing. ${ }^{7,8}$ Here, we used a common face emotion viewing paradigm to examine amygdala-PFC engagement to graded levels of specific face emotions. In addition to examining regional changes in neural activity, we assessed task-associated changes in amygdala connectivity. Irritability-related constructs have been associated with reduced functional connectivity between the amygdala and regulatory regions of the prefrontal cortex at rest in adults with high trait anger ${ }^{9}$ and during angry face emotion processing in adults with intermittent explosive disorder. ${ }^{10}$ Childhood generalized anxiety disorder, social phobia, separation anxiety disorder, and behavioral inhibition have been associated with disrupted amygdala-PFC functional connectivity, assessed while participants process face emotions. ${ }^{11-13}$ Indeed, one study in adult men found that high trait anxiety and anger interact to predict amygdala response to angry faces. ${ }^{14}$ However, to our knowledge, no study in youths has examined the effect on amygdala circuitry of anxiety and irritability, as either independent or interacting variables, although these symptoms often present together.

Our approach to sampling differs from most prior brain imaging work on anxiety or irritability. Specifically, we operationalized these symptoms dimensionally and studied them in children receiving psychiatric care. This differs from 2 common approaches in the literature. Some prior studies examined children with anxiety disorders, defined categorically, ${ }^{15}$ or 2 categories of irritability-related disorders, bipolar disorder and severe mood dysregulation or disruptive mood dysregulation disorder. ${ }^{16}$ These studies did not consider how symptoms of anxiety or irritability, occurring across disorders, relate to brain function. Other studies adopted continuous approaches, typically in community-based samples. ${ }^{17}$ These studies rarely included large numbers of youths surpassing clinical thresholds for a disorder. To address the limitations of these prior approaches, we studied the neural

\section{Key Points}

Question How does the brain respond to facial emotions signifying threat in youths with pathologic anxiety and/or irritability?

Findings In this functional magnetic resonance imaging study of 115 participants, anxiety and irritability were jointly associated with the amygdala's connectivity to regulatory regions in the prefrontal cortex during face emotion processing. In particular, when participants viewed very angry faces, high irritability and high anxiety were associated with increased amygdala-medial prefrontal cortex connectivity, while high irritability and low anxiety were associated with decreased connectivity in the same circuit.

Meaning Anxiety and irritability appear to interact to influence connectivity in the neural system mediating response to social threat.

correlates of dimensional measures of irritability and anxiety in youths undergoing treatment for clinically significant disorders.

In sum, we examined 115 youths with varying diagnoses and levels of anxiety and irritability using a common face emotion processing task. Based on prior studies that included either anxious or irritable youth (see also the eAppendix in the Supplement), we hypothesized that irritability and anxiety exhibit independent and interacting associations with perturbed amygdala-PFC function in response to specific face emotion displays. ${ }^{11-13,18-20}$

\section{Methods}

\section{Participants}

The study included 115 youths aged 8 to 17 years with primary diagnoses of disruptive mood dysregulation disorder (DMDD; $\mathrm{n}=37$ ), anxiety disorder (ANX; $\mathrm{n}=32$ ), attentiondeficit/hyperactivity disorder (ADHD; $n=24$ ), or no psychopathology (healthy volunteers; $n=22$ ) (Table 1 ; eTable 1 in the Supplement). Primary diagnosis reflected the chief symptom for which patients were seeking or receiving treatment. Consistent with an RDoC approach, the study recruited samples with diverse diagnoses and rich variability in symptom levels, particularly irritability and anxiety. While the chief symptom of youths with DMDD was severe irritability, they also had high rates of ANX (49\%) and ADHD (84\%). Because DMDD was exclusionary for the ANX or ADHD groups, patients in the latter 2 groups had low to moderate irritability. Data were obtained between November 2011 and July 2015. The National Institutes of Health institutional review board approved this study. Written consent/assent from parents/children was obtained, and youth were paid for participation.

The Affective Reactivity Index (ARI) ${ }^{23}$ and the Screen for Child Anxiety Related Disorders (SCARED) ${ }^{24}$ were used to measure irritability and anxiety, respectively. Data were collected within 60 days of scan and total scores for children and parents were averaged (see Figure 1 for distributions). See eMethods 1 in the Supplement for participant assessment and 


\begin{tabular}{|c|c|c|}
\hline Characteristic & Descriptive Statistics & $P$ Value \\
\hline \multicolumn{3}{|l|}{ Age, $y$} \\
\hline Mean (SD) & $13.2(2.6)$ & NA \\
\hline Range & $8-17$ & NA \\
\hline \multicolumn{3}{|l|}{ Sex } \\
\hline Male & 64 & NA \\
\hline Female & 51 & NA \\
\hline$I Q$, mean $(S D)^{a}$ & $110.2(13.4)$ & NA \\
\hline $\mathrm{SES}$, mean $(\mathrm{SD})^{\mathrm{b}}$ & $35.5(18.5)$ & NA \\
\hline \multicolumn{3}{|l|}{ ARI } \\
\hline Mean (SD) & $3.5(2.9)$ & \multirow[t]{2}{*}{ NA } \\
\hline Range & $0-12$ & \\
\hline \multicolumn{3}{|l|}{ SCARED } \\
\hline Mean (SD) & $18.6(12.5)$ & NA \\
\hline Range & $0-53.5$ & NA \\
\hline \multicolumn{3}{|c|}{ Presenting diagnosis, №. (\%) } \\
\hline None & $22(19)$ & NA \\
\hline Any anxiety & $32(28)$ & NA \\
\hline ADHD & $24(21)$ & NA \\
\hline DMDD & $37(32)$ & NA \\
\hline \multicolumn{3}{|c|}{ Lifetime diagnoses, №. (\%) } \\
\hline Any anxiety & $52(45)$ & NA \\
\hline ADHD & $58(50)$ & NA \\
\hline MDD & $7(6)$ & NA \\
\hline \multicolumn{3}{|l|}{ Medications, No. (\%) } \\
\hline SSRI & $11(10)$ & NA \\
\hline Stimulants & $40(35)$ & NA \\
\hline SGA & $14(12)$ & NA \\
\hline AED & $7(6)$ & NA \\
\hline \multicolumn{3}{|l|}{ Image quality, mean (SD) } \\
\hline Motionc & $0.077(0.045)$ & NA \\
\hline Censor fraction & $0.029(0.032)$ & NA \\
\hline \multicolumn{3}{|l|}{ Associations $^{d}$} \\
\hline ARI and SCARED & $r=0.43$ & $<.001$ \\
\hline ARI and age & $r=-0.26$ & .004 \\
\hline ARI and IQ & $r=0.06$ & .56 \\
\hline ARI and gender & $t=-0.19$ & .85 \\
\hline ARI and SES & $r=0.04$ & .73 \\
\hline ARI and motion & $r=0.25$ & .008 \\
\hline SCARED and age & $r=-0.22$ & .02 \\
\hline SCARED and IQ & $r=-0.04$ & .67 \\
\hline SCARED and SES & $r=0.10$ & .35 \\
\hline SCARED and gender & $t=-3.17$ & .002 \\
\hline SCARED and motion & $r=0.01$ & .91 \\
\hline
\end{tabular}

Abbreviations: ADHD, attention-deficit/hyperactivity disorder; AED, antiepileptic drug; ARI, Affective Reactivity Index: DMDD, disruptive mood dysregulation disorder; MDD, major depressive disorder; NA, not applicable; SCARED, Screen for Child Anxiety Related Disorders; SES, socioeconomic status; SGA, second-generation antipsychotic; SSRI, selective serotonin reuptake inhibitor.

a IQ was measured by the Wechsler Abbreviated Scale of Intelligence. ${ }^{21}$ IQ data were not available for 2 participants.

b SES was measured by the Hollingshead 2-factor index. ${ }^{22}$ These data were not available for 24 participants.

' Motion is calculated as the mean Euclidean distance of framewise volume shift after censoring.

${ }^{\mathrm{d}}$ All $d f$ values are 113, except in correlations with IQ and SES where they are 111 and 89, respectively. Correlations between dimensional measures have an acceptable tolerance in a linear model including ARI, SCARED, age, gender, motion, and the ARI by SCARED interaction (maximum variance inflation factor $=1.38$ ).
eTable 2 in the Supplement for participants excluded owing to poor or incomplete imaging data.

\section{Task}

An implicit face emotion processing task was adapted from Kim et al. ${ }^{25}$ Participants labeled the gender of 10 actors' happy, angry, and fearful face emotion pictures. ${ }^{26}$ Expressions at 50\%, $100 \%$, and $150 \%$ intensities were presented randomly for 2000 milliseconds followed by jittered fixation (mean, 1400 milli- seconds; range, 500-6000 milliseconds). Trials appeared in 3 blocks, generating 30 trials of each emotion at each intensity and 90 neutral face emotion trials.

\section{Imaging Procedures}

Magnetic resonance images (MRI) were acquired on a General Electric 3-T scanner with a 32-channel head coil. Blood oxygen level-dependent signal was measured by echoplanar imaging at $2.5 \times 2.5 \times 3.0-\mathrm{mm}$ voxel resolution. Standard pre- 
Figure 1. Distribution of Affective Reactivity Index and Screen for Child Anxiety Related Disorders Scores by Primary Diagnosis

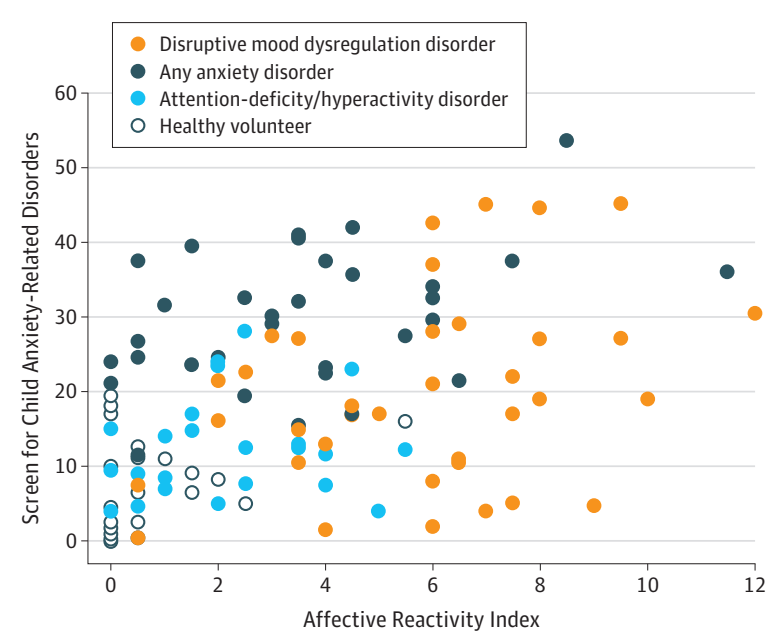

processing used FreeSurfer ${ }^{27}$ and Analysis of Functional Neuroimages (AFNI) ${ }^{28}$ software (eMethods 2 in the Supplement).

A general linear model estimated voxelwise blood oxygenation level-dependent signal change and generalized psychophysiological interaction ${ }^{29}$ for voxelwise functional connectivity of the AFNI DKD_Desai_MPM ${ }^{30}$ atlas-defined amygdala (eMethods 3 in the Supplement).

\section{Statistical Analyses}

Analyses conducted between August 2015 and August 2016 used AFNI and R (R Foundation for Statistical Computing). ${ }^{31}$ Omnibus analyses used mixed-effects models in AFNI's $3 \mathrm{dLME}^{32}$ for images and the R package $\operatorname{lme} 4^{33}$ for behavior and post hoc analyses of imaging results. The mixed model tested effects of emotion, intensity, ARI, and SCARED, with age and gender as covariates and participant as a random effect. Motion was an additional covariate in all imaging analyses. Emotion and intensity were modeled as within-participant factors, each with 3 levels (emotion: happy, angry, and fearful; intensity: $50 \%, 100 \%$, and $150 \%$ ). Continuous variables were mean centered. Table 1 shows the associations among variables. Dependent variables were accuracy (percentage correct gender identification) and mean reaction time for behavioral analyses, and neural activity or amygdala connectivity for imaging analyses. Only trials with accurate gender identification were included. Responses to neutral faces served as a positive control (eFigure 1 in the Supplement).

The imaging analysis was conducted across a wholebrain mask, including only voxels where data existed for $90 \%$ or more of participants. The voxelwise $P$ value threshold was .001 , with multiple testing correction to $\mathrm{a}=.05$ via Monte Carlo cluster-size simulation with a gaussian plus exponential spatial autocorrelation function to estimate smoothness (AFNI's 3dClustSim). We applied Bonferroni adjustment for 3 tests (1 neural activity and 2 generalized psychophysiological interactions) resulting in $a=.05 / 3=0.0167$ and cluster size greater than 42 , reported with size ( $k$ ) and center-of-mass (CoM) coordinates in Talairach space. Additional event-specific analyses relied on mean connectivity or activity extracted via AFNI's 3dROIstat.

For post hoc analyses, we fit mixed-effects models using the same formula as the functional MRI group analysis mixed model. From these, we used general linear tests (Wald $x^{2}$ ) of specific contrasts or fixed effects of any variables while adjusting for all others (R package phia ${ }^{34}$ ). We used HolmBonferroni corrections for multiple comparisons. Participants with influential observations were identified by their Cook's distance using R package influence.ME. ${ }^{35}$ Influential observations were participants with a Cook's distance greater than 0.053, a threshold defined by sample size and number of mixed-model parameters $(n=39) .{ }^{36}$ Iterative post hoc analyses leaving out individuals taking each class of medication, or who were influential, were done to ensure findings were robust to their exclusion (medication classes are listed in Table 1).

\section{Results}

\section{Behavior}

Accuracy was associated with irritability as a function of emotion and intensity (ARI by emotion by intensity interaction; $F_{4,888}=2.77 ; P=.03$; eFigure 2 in the Supplement). Specifically, increasing irritability was associated with decreasing accuracy when labeling the gender of angry faces at $100 \%$ (Wald $X_{1}^{2}=7.58 ; P=.05$; age- and sex-adjusted ARI and accuracy, $r=-0.27 ; P=.004$ ) and $150 \%$ (Wald $\chi^{2}{ }_{1}=11.94 ; P=.005$; ageand gender adjusted ARI and accuracy, $r=-0.31 ; P<.001)$. Imaging analyses controlled for this potential confound by including only correct trials. There were no associations between accuracy and SCARED or between mean correct reaction time and either irritability or anxiety.

\section{Amygdala Functional Connectivity}

Connectivity between the left amygdala and left medial PFC interacted with all modeled terms of interest (ARI by SCARED by emotion by intensity; $F_{4,888}=9.20 ; P<.001 ; k=61$; $\mathrm{CoM}=-7.5,58.1,10.1$ ) (Figure 2). Post hoc general linear tests revealed a relatively clear pattern of results. The association between ARI scores and change in connectivity when viewing high-intensity (150\%) angry faces varied significantly with SCARED score (at 150\% angry; Wald $\chi^{2}{ }_{1}=21.3 ; P<.001$ ). Figure $2 \mathrm{~B}$ and $\mathrm{C}$ illustrate the interaction, showing a decrease in connectivity in participants who are highly anxious and irritable (blue quadrant; Figure 2B), but an increase in connectivity in those who are highly anxious but not irritable (red quadrant; Figure 2B).

In addition, a lower-level interaction between the left amygdala and the left lateral orbitofrontal cortex emerged (ARI by SCARED by emotion; $F_{2,888}=15.28 ; P<.001 ; k=52$; $\mathrm{CoM}=-32.1,33.1,-5)$. Relative to angry expressions, connectivity to fearful expressions decreased in highly irritable, lowanxious individuals (eFigure 3 in the Supplement). Finally, a main effect of SCARED on left amygdala connectivity was present in the subgenual anterior cingulate/orbitofrontal cortex $\left(F_{1,108}=25.48 ; P<.001 ; k=43 ; \mathrm{CoM}=-15.9,33.1,-3.8\right)$, where 
Figure 2. Left Amygdala Functional Connectivity During Implicit Processing of $150 \%$ Angry Face Emotions

A Whole-brain analysis

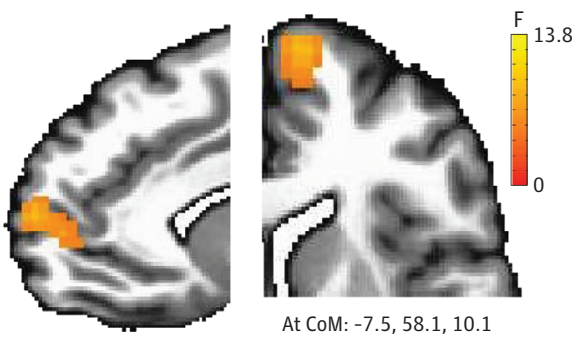

B Connectivity

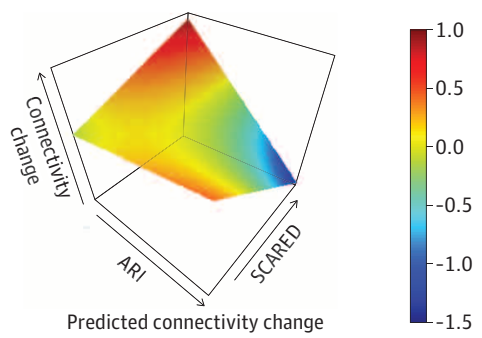

C Variability
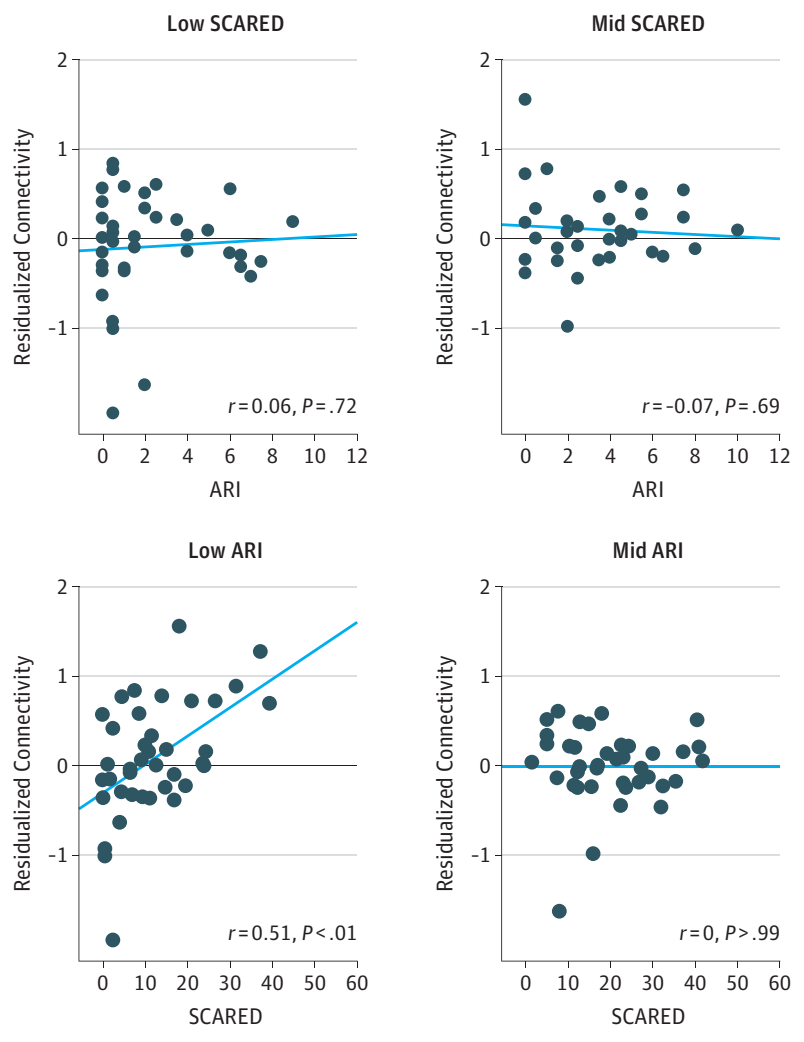

SCARED was positively associated with connectivity (age-, gender-, and motion-adjusted $r=0.37 ; P<.001$; eFigure 3 in the Supplement).

Connectivity to the right amygdala was modulated by SCARED and intensity in the bilateral superior temporal gyri (SCARED by intensity; right: $F_{2,888}=15.03 ; P<.001 ; k=95$; CoM $=61.2,-6.2,3.8$; and left: $F_{2,888}=13.00 ; P<.001 ; k=69$; $\mathrm{CoM}=-53.8,-23.8,8.8)$. In both areas, SCARED was associated with the difference in connectivity between $50 \%$ and both $100 \%$ and $150 \%$ intensities across emotions (Wald $\chi_{1}^{2} \geq 18$; all $P<$.001). Generally, this difference increased with increasing SCARED (age-, gender-, and motion-adjusted $r>0.28$; all $P<.003)$.

\section{Activation}

Activation was associated with irritability rather than anxiety. Across intensities, 7 regions exhibited an ARI-
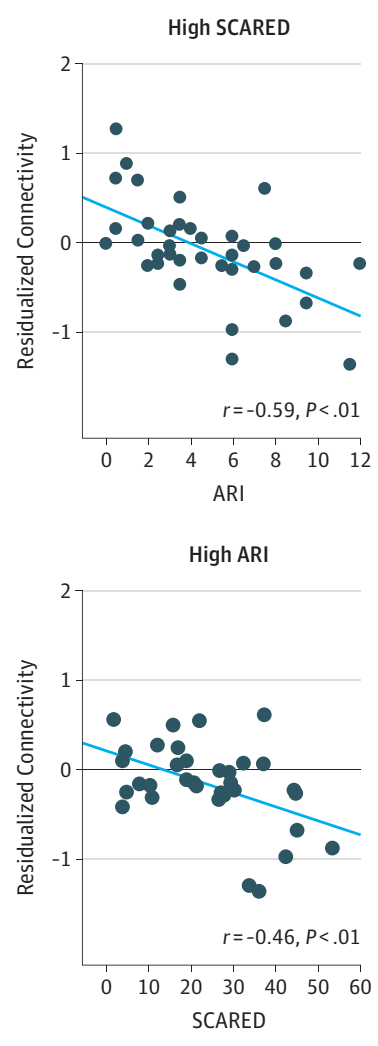
neural activity.

\section{Post Hoc Analyses}

A, Results of the whole-brain analysis of left amygdala functional connectivity. In functional connectivity to the amygdala, a medial prefrontal cortex (mPFC) region showed an interaction among Affective Reactivity Index (ARI), Screen for Child Anxiety Related Disorders (SCARED), emotion, and intensity. B, Associations among ARI, SCARED, and connectivity driving this interaction. From the MPFC region in each patient, we extracted mean voxelwise change in connectivity for each condition (the psychophysiologic interaction coefficients). The change in connectivity is relative to baseline connectivity across the task, modeled at the single-patient level. ${ }^{29} \mathrm{We}$ entered these values in the same mixed-effects model as in the main analysis and determined that the effect of ARI and SCARED had significant interactive effects only at the $150 \%$ angry face condition. For this condition, the predicted change in connectivity from the fitted mixed model is shown on the left (age at center, 13.2 years; female; ARI range, 0-12; SCARED range, 0-54). Relative to baseline amygdala-mPFC connectivity, connectivity decreases during implicit processing of $150 \%$ angry faces for highly irritable and anxious individuals. C, Graphs depict variability. We partialled out the effects of motion, age, and gender across task conditions from mean change in connectivity. We plotted the resultant residual change in connectivity for $150 \%$ angry faces against ARI or SCARED for individuals grouped into tertiles of SCARED or ARI scores, respectively. Descriptive statistics are given for the plotted data. CoM indicates center of mass.

by-emotion interaction (Table 2; eFigure 4 in the Supplement). This generally reflected increasing activity with increasing irritability to happy or angry, relative to fearful, faces (Table 2). No associations manifested between SCARED and

In leave-out analyses, we evaluated confounding by medication status (eTable 3 in the Supplement). We iteratively excluded individuals by medication class in analyses of mean connectivity or activity. The 4 participants whose medication status was unknown were excluded from these analyses. All $F$ tests of the effects we found in the whole sample remained statistically significant, with a similar pattern of significant post hoc contrasts, except in the right fusiform gyrus where, when patients receiving antipsychotics were excluded, the $F$ test became a trend $\left(F_{2,744}=2.3 ; P=.10\right)$. To facilitate comparisons 


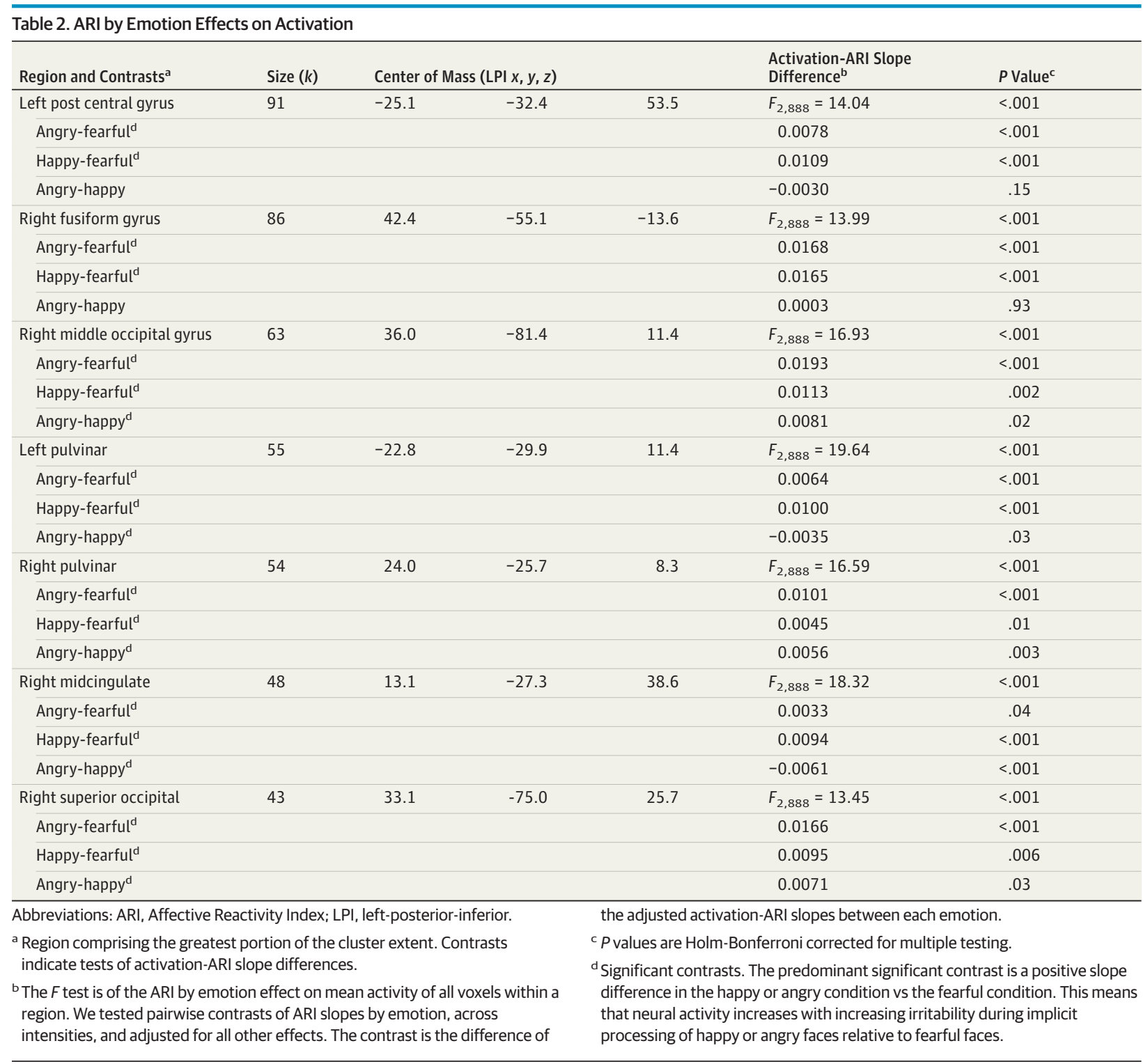

with prior research, categorical analyses of diagnosis are presented (eResults 1, eResults 2, and eResults 3 in the Supplement).

\section{Discussion}

Two key findings from this study clarify associations among irritability, anxiety, and neural function. First, during implicit processing of emotional faces, connectivity between the amygdala and its prefrontal regulatory areas varied strongly as a function of both irritability and anxiety, across healthy youth and those with at least 1 of 3 diagnoses (anxiety disorder, DMDD, or ADHD). Specifically, when participants viewed intensely angry expressions, high levels of both anxiety and irritability were associated with decreased amygdala-medial prefrontal cortex connectivity, whereas high levels of anxiety but low levels of irritability were associated with increased con- nectivity. Second, for regional activation, more findings emerged for irritability than for dimensional measures of anxiety or for categorical diagnoses. Specifically, high levels of irritability were associated with brain function as well as to task performance, particularly when labeling the gender of intensely angry faces.

Several factors suggest the robust nature of our findings. Our relatively large sample of well-characterized children showed high variability for both anxiety and irritability, with many youths exhibiting symptoms well within the clinical range. This maximized statistical power to examine associations between brain function and clinically meaningful variation in these 2 symptom dimensions. Moreover, we used a relatively conservative analytic strategy, with an omnibus statistical model and appropriate whole-brain-corrected statistical thresholds for tests of high-order interactions. (See eResults 4 in the Supplement for resampling-based tests of robustness.) The use of an event-related design with face- 
morphing procedures allowed us to control for performance confounds while linking specific clinical profiles to brain functions engaged by specific stimuli. Observed associations manifested with medium to large effect sizes in regions previously implicated in emotional processes, including face emotion perception.

Our findings meaningfully extend data on pediatric irritability. On this implicit face emotion processing task, most findings were associated with irritability rather than anxiety, largely in responses to angry faces. Behaviorally, increased irritability predicted decreased gender-labeling accuracy for intensely angry faces, suggesting that such faces are distracting to irritable youths. In several brain regions, particularly in the ventral visual stream and pulvinar, increased irritability predicted increased neural activity in response to angry and/or happy, relative to fearful, faces. Angry faces represent a social threat and are particularly salient to individuals prone to irritability, anger, and reactive aggression ${ }^{4,6,37,38}$; happiness, like anger, is an expression that can result in approach behavior. Our findings are consistent with prior studies linking irritability to responses to happy and angry faces in the ventral stream ${ }^{20,39,40}$ and to aberrant neural responses to a range of face emotions in visual and medial temporal regions. ${ }^{18-20,39,40}$

Importantly, our findings extend prior research suggesting that angry faces disrupt amygdala-PFC connectivity ${ }^{10}$ and reduce medial PFC activity ${ }^{37}$ in aggressive individuals. Specifically, the current findings indicate that co-occurring anxiety modulates amygdala-medial PFC connectivity in irritable youths. This suggests that youths with high levels of irritability and anxiety represent a meaningful subgroup in terms of brain function. Future research might consider whether this subgroup also exhibits distinct longitudinal clinical trajectories and responses to treatment.

However, some of our findings for irritability did not replicate previous work. For example, the current study did not detect associations between irritability-associated neural responses and DSM diagnosis. In contrast, using a different study design and analytic approach, Wiggins et $\mathrm{al}^{2 \mathrm{O}}$ reported that the neural correlates of irritability during explicit face emotion labeling differ between bipolar disorder and DMDD. Other studies revealed that severe mood dysregulation, a phenotype similar to DMDD, predicted activation profiles on implicit faceviewing tasks independent of degree of irritability. ${ }^{18,39}$ Future studies might consider whether such inconsistent findings reflect imaging methods, classification approaches, or sampling characteristics.

Our findings also extend previous research in anxiety. Compared with prior reports, we examined a relatively large number of symptomatic, medication-free youths seeking treatment for an anxiety disorder. Both this feature and our use of a continuous measure to characterize anxiety increased statistical power. Our finding of an association among high anxiety, low irritability, and relatively high amygdala-PFC connectivity is consistent with some studies that examined associations between amygdala-PFC connectivity and anxiety alone. ${ }^{11-13}$ However, while our findings replicate such prior work on amygdala-PFC connectivity, we did not replicate prior activation findings, ${ }^{39}$ perhaps because of our choice of task (eResults 1 in the Supplement). Regardless, the findings that did emerge for connectivity suggest that aberrant amygdala-PFC connectivity represents one of the few replicable associations among many inconsistent findings in research on anxiety using implicit face emotion viewing tasks. Of note, recent data suggest that connectivity measures may be more stable than activation measures. ${ }^{41}$ This may be relevant, not only to prior reports on anxiety, but also to our current findings, which are more robust for connectivity than for activation.

\section{Limitations}

This study had limitations. The cross-sectional design of this study was a fundamental limitation. These results apply to irritability and anxiety only in the disorders that are wellsampled in this study. They do not apply to other diagnostic groups where high irritability and anxiety are often present (eg, major depressive disorder or bipolar disorder); such groups should be included in future studies. Inclusion criteria varied somewhat across diagnoses. Thus, all patients with anxiety disorders in the absence of DMDD or ADHD were actively seeking treatment, whereas most patients with DMDD or ADHD were already receiving treatment. The fact that associations with symptom dimensions manifested independent of diagnostic group suggests that this limitation does not account for our findings. Differences in psychotropic medication exposure may have influenced the results, although post hoc analyses suggest that no specific medication class explained the findings. Severely irritable children typically receive complex medication regimens, and the severity of their illness makes it unethical to maintain and study such youths medicationfree. Given the stability of the $\mathrm{ARI}^{23}$ and SCARED ${ }^{42}$ and to include as many participants as possible, we allowed up to 60 days between completion of scales and scan date, although $59 \%$ of participants were scanned within 10 days of scale completion. This time lag may have made our measurement of irritability and anxiety less precise. Finally, by using an amygdala seed based on a probabilistic atlas, the findings may reflect signal from surrounding structures in some individuals. However, in post hoc analyses, connectivity results were confirmed using each individual's FreeSurfer-parcellated amygdala.

\section{Conclusions}

We examined associations among neural connectivity, activity, and dimensional measures of 2 commonly co-occurring symptoms in youths, irritability and anxiety, across disorders that often present to clinicians. We found that these 2 prominent dimensions of pediatric psychopathology have interactive, rather than additive, effects on pathophysiology when patients process social threat. This could suggest the need for clinicians to attend to the co-occurrence of anxiety and irritability because the presence of both symptoms might have a unique effect on a child's response to social threat and/or to treatment, including psychotherapeutic treatments focused on 
social interactions. These findings also have implications for both clinicians and researchers interested in the RDoC framework because they suggest that, like comorbidity among DSM-5 diagnoses, co-occurrence of RDoC traits has important pathophysiological implications that might ultimately affect psychiatric diagnosis.

\section{ARTICLE INFORMATION}

Accepted for Publication: October 7, 2016.

Published Online: November 30, 2016 doi:10.1001/jamapsychiatry.2016.3282

Author Affiliations: Division of Child and Adolescent Psychiatry, Department of Psychiatry, University of Colorado, Anshutz Medical Campus Aurora (Stoddard); Section on Bipolar Spectrum Disorders, Emotion and Development Branch, National Institute of Mental Health, National Institutes of Health, Department of Health and Human Services, Bethesda, Maryland (Stoddard, Tseng, Yi, Donahue, Brotman, Towbin, Leibenluft); Department of Psychology, University of Denver, Denver, Colorado (Kim); Scientific and Statistical Computing Core, National Institute of Menta Health, National Institutes of Health, Department of Health and Human Services, Bethesda, Maryland (Chen); currently with Department of Psychology and Neuroscience, University of North Carolina at Chapel Hill (Yi); Section on Development and Affective Neuroscience, Emotion and Development Branch, National Institute of Mental Health, National Institutes of Health, Department of Health and Human Services, Bethesda, Maryland (Pine).

Author Contributions: Drs Stoddard and Leibenluft had full access to all the data in the study and take responsibility for the integrity of the data and the accuracy of the data analysis. Concept and design: Kim, Brotman, Pine, Leibenluft. Acquisition, analysis, or interpretation of data: Stoddard, Tseng, Chen, Yi, Donahue, Brotman, Towbin, Pine, Leibenluft.

Drafting of the manuscript: Stoddard, Brotman, Pine, Leibenluft.

Critical revision of the manuscript for important intellectual content: Tseng, Kim, Chen, Yi, Donahue, Brotman, Towbin, Pine.

Statistical analysis: Stoddard, Tseng, Chen, Pine. Administrative, technical, or material support Donahue, Brotman, Towbin, Pine, Leibenluft.

Conflict of Interest Disclosures: None reported.

Funding/Support: This research was supported by the Intramural Research Program of the National Institute of Mental Health, and it was conducted under protocols 02-M-0021 (ClinicalTrials.gov Identifier NCT00025935), 00-M-0198 (NCT00006177), and 01-M-0192 (NCT00018057).

Role of the Funder/Sponsor: The funder had no role in the design and conduct of the study; collection, management, analysis, and interpretation of the data; preparation or review of the manuscript; and decision to submit the manuscript for publication but had a role in the approval of the manuscript.

Additional Contributions: We thank the participants and families as well as the staff of the Emotion and Development Branch and the Scientific and Statistical Computing Core at the National Institute of Mental Health. In particular, we thank Dan Barlow, BS, Emotion and Development Branch, for programming support and Rick Reynolds, MS, Scientific and Statistical Computing Core, for imaging statistics guidance. Mr Barlow and
Mr Reynolds' contributions were paid for by their respective departments.

Additional Information: This research used the computational resources of the National Institutes of Health High-Performance Computing Biowulf cluster (http://hpc.nih.gov).

\section{REFERENCES}

1. Stringaris A, Goodman R. Longitudinal outcome of youth oppositionality: irritable, headstrong, and hurtful behaviors have distinctive predictions. J Am Acad Child Adolesc Psychiatry. 2009;48(4):404-412.

2. Brotman MA, Schmajuk M, Rich BA, et al. Prevalence, clinical correlates, and longitudinal course of severe mood dysregulation in children. Biol Psychiatry. 2006;60(9):991-997.

3. Savage J, Verhulst B, Copeland W, Althoff RR, Lichtenstein P, Roberson-Nay R. A genetically informed study of the longitudinal relation between irritability and anxious/depressed symptoms. J Am Acad Child Adolesc Psychiatry. 2015;54(5):377-384.

4. Stoddard J, Sharif-Askary B, Harkins EA, et al. An open pilot study of training hostile interpretation bias to treat disruptive mood dysregulation disorder. J Child Adolesc Psychopharmacol. 2016;26(1):49-57.

5. Bar-Haim Y, Lamy D, Pergamin L, Bakermans-Kranenburg MJ, van IJzendoorn MH Threat-related attentional bias in anxious and nonanxious individuals: a meta-analytic study. Psychol Bull. 2007;133(1):1-24.

6. Hommer RE, Meyer A, Stoddard J, et al. Attention bias to threat faces in severe mood dysregulation. Depress Anxiety. 2014;31(7):559-565.

7. Leibenluft E, Stoddard J. The developmental psychopathology of irritability. Dev Psychopathol. 2013;25(4, pt 2):1473-1487.

8. Kim MJ, Loucks RA, Palmer AL, et al. The structural and functional connectivity of the amygdala: from normal emotion to pathological anxiety. Behav Brain Res. 2011;223(2):403-410.

9. Fulwiler CE, King JA, Zhang N.

Amygdala-orbitofrontal resting-state functional connectivity is associated with trait anger. Neuroreport. 2012;23(10):606-610.

10. Coccaro EF, McCloskey MS, Fitzgerald DA Phan KL. Amygdala and orbitofrontal reactivity to social threat in individuals with impulsive aggression. Biol Psychiatry. 2007;62(2):168-178.

11. Hardee JE, Benson BE, Bar-Haim Y, et al. Patterns of neural connectivity during an attention bias task moderate associations between early childhood temperament and internalizing symptoms in young adulthood. Biol Psychiatry. 2013;74(4):273-279.

12. Gold AL, Shechner T, Farber MJ, et al. Amygdala-cortical connectivity: associations with anxiety, development, and threat. Depress Anxiety. 2016;33(10):917-926.

13. Monk CS, Telzer EH, Mogg K, et al. Amygdala and ventrolateral prefrontal cortex activation to masked angry faces in children and adolescents with generalized anxiety disorder. Arch Gen Psychiatry. 2008;65(5):568-576.

14. Carré JM, Fisher PM, Manuck SB, Hariri AR. Interaction between trait anxiety and trait anger predict amygdala reactivity to angry facial expressions in men but not women. Soc Cogn Affect Neurosci. 2012;7(2):213-221.

15. Beesdo K, Lau JY, Guyer AE, et al. Common and distinct amygdala-function perturbations in depressed vs anxious adolescents. Arch Gen Psychiatry. 2009;66(3):275-285.

16. Leibenluft E. Severe mood dysregulation, irritability, and the diagnostic boundaries of bipolar disorder in youths. Am J Psychiatry. 2011;168(2): 129-142.

17. Qin S, Young CB, Duan X, Chen T, Supekar K, Menon V. Amygdala subregional structure and intrinsic functional connectivity predicts individual differences in anxiety during early childhood. Biol Psychiatry. 2014;75(11):892-900.

18. Thomas LA, Brotman MA, Muhrer EJ, et al. Parametric modulation of neural activity by emotion in youth with bipolar disorder, youth with severe mood dysregulation, and healthy volunteers. Arch Gen Psychiatry. 2012;69(12): 1257-1266.

19. Thomas LA, Kim P, Bones BL, et al. Elevated amygdala responses to emotional faces in youths with chronic irritability or bipolar disorder. Neuroimage Clin. 2013;2:637-645.

20. Wiggins JL, Brotman MA, Adleman NE, et al. Neural correlates of irritability in disruptive mood dysregulation and bipolar disorders. Am J Psychiatry. 2016;173(7):722-730.

21. Wechsler D. WASI Manual. San Antonio, TX: Psychological Corp; 1999.

22. Hollingshead AB. Two Factor Index of Social Position. Mimeo. New Haven, CT: Yale University; 1957.

23. Stringaris A, Goodman R, Ferdinando S, et al. The Affective Reactivity Index: a concise irritability scale for clinical and research settings. $J$ Child Psychol Psychiatry. 2012;53(11):1109-1117.

24. Birmaher B, Brent DA, Chiappetta L, Bridge J, Monga S, Baugher M. Psychometric properties of the Screen for Child Anxiety Related Emotional Disorders (SCARED): a replication study. J Am Acad Child Adolesc Psychiatry. 1999;38(10):1230-1236.

25. Kim P, Thomas LA, Rosen BH, et al. Differing amygdala responses to facial expressions in children and adults with bipolar disorder. Am J Psychiatry. 2012;169(6):642-649.

26. Ekman P, Friesen WV. Pictures of Facial Affect. Palo Alto, CA: Consulting Psychologists Press; 1976.

27. Ségonne F, Dale AM, Busa E, et al. A hybrid approach to the skull stripping problem in MRI. Neuroimage. 2004;22(3):1060-1075.

28. Cox RW. AFNI: software for analysis and visualization of functional magnetic resonance neuroimages. Comput Biomed Res. 1996;29(3): 162-173. 
29. McLaren DG, Ries ML, Xu G, Johnson SC. A generalized form of context-dependent psychophysiological interactions (gPPI): a comparison to standard approaches. Neuroimage. 2012;61(4):1277-1286

30. Desikan RS, Ségonne F, Fischl B, et al. An automated labeling system for subdividing the human cerebral cortex on MRI scans into gyral based regions of interest. Neuroimage. 2006;31(3): 968-980.

31. $R$ : A language and environment for statistical computing [computer program]. Version 3.2.3 Vienna, Austria: R Foundation for Statistical Computing; 2015.

32. Chen G, Saad ZS, Britton JC, Pine DS, Cox RW. Linear mixed-effects modeling approach to FMRI group analysis. Neuroimage. 2013;73:176-190.

33. Bates D, Maechler M, Bolker B, Walker S. Fitting linear mixed-effects models using Ime4. J Stat Softw. 2015;67(1):1-48.
34. De Rosario-Martinez H. phia: Post-Hoc Interaction Analysis [computer program]. Version 0.2-1. R package.2015

35. Nieuwenhuis R, Te Grotenhuis M, Pelzer $B$. Influence.ME: tools for detecting influential data in mixed effects models. $R$ J. 2012;4(2):38-47.

36. Fox J. Regression Diagnostics: An Introduction. Sage University Paper Series on Quantitative Applications in the Social Sciences, 07-079. Newbury Park, CA: Sage. 1991.

37. Beyer F, Münte TF, Göttlich M, Krämer UM Orbitofrontal cortex reactivity to angry facial expression in a social interaction correlates with aggressive behavior. Cereb Cortex. 2015;25(9): 3057-3063.

38. Veenstra L, Schneider IK, Bushman BJ, Koole SL. Drawn to danger: trait anger predicts automatic approach behaviour to angry faces [Published online February 19, 2016]. Cogn Emot. doi:10.1080 /02699931.2016.1150256
39. Thomas LA, Brotman $M A$, Bones $B L$, et al. Neural circuitry of masked emotional face processing in youth with bipolar disorder, severe mood dysregulation, and healthy volunteers. Dev Cogn Neurosci. 2014;8:110-120.

40. Tseng WL, Thomas LA, Harkins E, Pine DS, Leibenluft E, Brotman MA. Neural correlates of masked and unmasked face emotion processing in youth with severe mood dysregulation. Soc Cogn Affect Neurosci. 2016;11(1):78-88.

41. White LK, Britton JC, Sequeira S, et al. Behavioral and neural stability of attention bias to threat in healthy adolescents. Neuroimage. 2016; 136:84-93.

42. Hale WW III, Raaijmakers $Q$, Muris $P$ van Hoof A, Meeus W. Developmental trajectories of adolescent anxiety disorder symptoms: a 5-year prospective community study. J Am Acad Child Adolesc Psychiatry. 2008;47(5):556-564. 